\section{Rationale for treating Huntington's with a sirolimus and lithium combination}

A promising treatment approach to Huntington's disease is promotion of autophagy to dispose of aggregate-prone proteins such as mutant huntingtin. Lithium has been previously shown to promote autophagy through inhibition of the enzyme inositol monophosphatase (IMPase). A new study by Sarkar et al. demonstrates, however, that another enzyme inhibited by lithium, glycogen synthase kinase-3 $\beta$ (GSK3 $\beta$ ), suppresses autophagy by activating the mammalian target of rapamycin (mTOR). The authors show that this inhibitory effect on autophagy might be counteracted by administering lithium in combination with the mTOR inhibitor sirolimus (rapamycin).

In vitro, treatment with lithium in combination with sirolimus increased the number of autophagosomes more than did either treatment alone $(P<0.05)$, whereas the effect of the combination treatment on mTOR activity was similar to that of sirolimus alone. This finding agrees with previous observations in cell culture that the upregulation of autophagy results from both mTOR inhibition by sirolimus and IMPase inhibition by lithium. Sarkar et al. showed that the activating effect of GSK3 $\beta$ on mTOR was nullified by downstream inhibition of mTOR by sirolimus. Supporting this concept, in a Drosophila model of Huntington's disease, treatment with either lithium or sirolimus alone protected against neurodegeneration compared with control, but the effect of both compounds in combination was significantly greater $(P<0.05$ vs either treatment alone).

The authors conclude that sirolimus and lithium act through two independent pathways to generate an additive positive effect on autophagy. The study shows clear added benefit from combining these two treatments in cell and animal models of Huntington's disease.

Original article Sarkar S et al. (2007) A rational mechanism for combination treatment of Huntington's disease using lithium and rapamycin. Hum Mol Genet 17: 170-178

\section{Sativex ${ }^{\circledR}$ improves pain control in patients with unresponsive and persistent neuropathic pain}

Chronic neuropathic pain is treatable by local anesthetics, antidepressants, antiepileptic drugs and opioids, but unresponsiveness and treatment resistance are common. According to a new study, better pain control might be achieved by adding Sativex ${ }^{\circledR}$ (GW Pharmaceuticals, Salisbury, UK) to the existing stable analgesia regime. This drug, derived from extracts of selected strains of cannabis plants (Cannabis sativa), contains the active cannabinoids delta-9-tetrahydrocannabinol (THC) and cannabidiol (CBD), and can be administered as a sublingual and oropharyngeal spray.

Nurmikko et al. performed a randomized, double-blind, placebo-controlled trial to evaluate oromucosally administered Sativex ${ }^{\circledR}$ in 125 patients with neuropathic pain of peripheral origin, over a 5-week period. The patients all had unresponsive and persistent pain despite therapy. In total, 63 patients were assigned Sative ${ }^{\circledR}$ and 62 were assigned a placebo spray: patients self-administered their spray, with a maximum of 48 doses allowed in $24 \mathrm{~h}$, and all remained on their existing stable analgesia during the trial.

Among those receiving Sativex ${ }^{\circledR}, 26 \%$ reported a reduction in pain intensity of $>30 \%$, which is generally considered as clinically meaningful. Only $15 \%$ of patients taking placebo reported a reduction of $>30 \%$. Patients taking Sativex ${ }^{\circledR}$ also showed significant reductions in allodynia and disability and an improvement in sleep patterns compared with patients receiving placebo.

The authors conclude that Sativex ${ }^{\circledR}$ can provide effective relief of peripheral neuropathic pain when given in addition to existing medication. They noted that the results were particularly encouraging given the refractory nature of the pain among the patients in their study group.

Original article Nurmikko TJ et al. (2007) Sativex successfully treats neuropathic pain characterised by allodynia: a randomised, double-blind, placebo-controlled clinical trial. Pain 133: 210-220

\section{Serum glial fibrillary acidic protein-a diagnostic biomarker for glioblastoma multiforme}

Currently, a definite diagnosis of glioblastoma multiforme (GBM) can be made only by the histopathological analysis of tumor tissue. Glial fibrillary acidic protein (GFAP) is a marker of astrocytic differentiation and is expressed in astroglial tumors. On the basis of the hypothesis 